\title{
Ultimate High Conductivity of Multilayer Graphene Examined by Multiprobe Scanning Tunneling Potentiometry on Artificially Grown High-Quality Graphite Thin Film
}

Hiroyuki Mogi, ${ }^{\dagger}$ Takafumi Bamba, ${ }^{\dagger}$ Mutsuaki Murakami, ${ }^{\ddagger}$ Yuki Kawashima, ${ }^{\ddagger}$ Masamichi Yoshimura, ${ }^{\S}$ Atsushi Taninaka, ${ }^{\dagger}$ Shoji Yoshida, ${ }^{\dagger}$ Osamu Takeuchi, ${ }^{\dagger}$ Haruhiro Oigawa, ${ }^{\dagger}$ and Hidemi Shigekawa ${ }^{*}{ }^{\dagger}$ (0)

${ }^{\dagger}$ Faculty of Pure and Applied Sciences, University of Tsukuba, Tsukuba 305-8573, Japan

${ }^{\ddagger}$ Materials Solutions New Research Engine, KANEKA Corporation, Osaka 566-0072, Japan

${ }^{\S}$ Graduate School of Engineering, Toyota Technological Institute, Nagoya 466-8511, Japan

Supporting Information

\begin{abstract}
With the discovery of graphene, the excellent characteristics of graphite (multilayer graphene), such as high thermal and electric conductivities, heat resistance, and chemical stability, have also been attracting considerable attention again. However, the production of a large-area $\left(>0.1 \times 0.1 \mathrm{~m}^{2}\right)$ graphite thin film with uniformly high electric conductivity, which will be indispensable in industrial applications, has been difficult because of its breakage occurring during the growth process. Recently, we have succeeded, for the first time, in growing large-area highquality graphite films with thicknesses from $\sim 0.5$ to $\sim 3 \mu \mathrm{m}$ by an industrial method, providing a fundamental platform for examining and utilizing the intrinsic and ultimate characteristics of such a material. Here, as a step toward realizing such efforts, we performed microscopic measurements of electric conductivity by the method of multiprobe
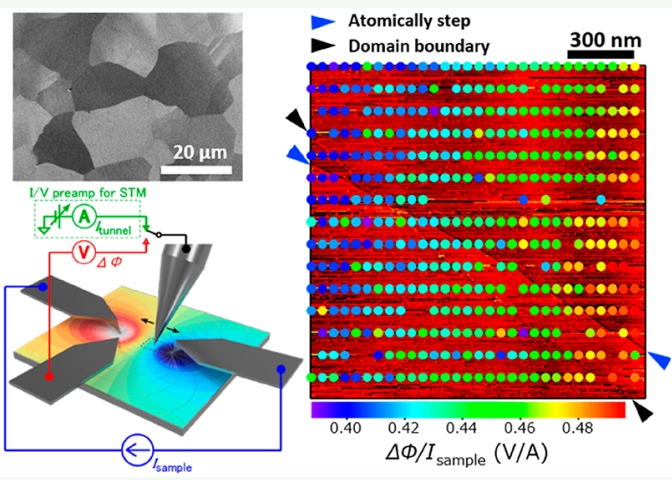
scanning tunneling potentiometry we have developed. Using the resistivity along the $c$-axis, $\rho_{c}=1.72 \pm 0.04 \mathrm{~m} \Omega \cdot \mathrm{m}$, which was directly measured for the first time, we determined the resistivity in the $a b$ plane, $\rho_{a b}$, to be $0.30 \pm 0.01 \mu \Omega \cdot \mathrm{m}$, indicating that the conductance of the graphite thin film we grew is about 1.3 times the previous highest reported value for the highest grade single-crystal natural graphite with an ideal structure without grain boundaries or wrinkles $(0.38 \mu \Omega \cdot \mathrm{m})$. Both the advantage of multilayer graphene, which is considered to reduce the effect of grain boundaries on conductance, and the decrease in conductance as an effect of wrinkles were directly evaluated.
\end{abstract}

KEYWORDS: multilayer graphene, artificially grown graphite, high conductivity, multi probe, scanning tunneling potentiometry

\section{INTRODUCTION}

Graphite is a material that has been used for a long time because of its excellent properties such as high electric and thermal conductivities, chemical stability, heat resistance, and low specific gravity. For example, the carrier mobility in the $a b$ plane of graphite is much larger than that of metals (for example: $\mathrm{Cu}, 16 \mathrm{~cm}^{2} /(\mathrm{V} \cdot \mathrm{s})$; graphite basal plane, $\sim 10000$ $\left.\mathrm{cm}^{2} /(\mathrm{V} \cdot \mathrm{s})\right)$. Thereby, the electric conductivity of graphite in the $a b$-plane, $\sim 25000 \mathrm{~S} / \mathrm{cm}$, is extremely high for a nonmetallic material. With the discovery of graphene, ${ }^{1}$ further applications of graphite that utilize its outstanding properties as multilayer graphene, for example, as fabricable electrode materials for solar cells, ${ }^{2}$ light-emitting diodes, ${ }^{3}$ flat panel displays, ${ }^{4}$ smart windows, ${ }^{5}$ touch panels, ${ }^{6}$ and Li-ion batteries, ${ }^{7}$ in supercapacitors, ${ }^{8,9}$ and as a superconductor, ${ }^{10-12}$ have been actively studied. For a field-effect transistor (FET) channel formed by several layers of $\mathrm{MoS}_{2}$ with a multilayer graphene electrode prepared by peeling layers from a bulk graphite, a high mobility exceeding $10^{4} \mathrm{~cm}^{2} /(\mathrm{V} \cdot \mathrm{s})$ was achieved. ${ }^{13}$ To advance these applications, it is highly desirable to develop a method of industrially obtaining high-quality thin-film graphite with a large area.

Kish graphite and highly oriented pyrolytic graphite (HOPG) are well-known artificial graphite materials, which are produced from the liquid phase (molten metal) and the gas phase, respectively. ${ }^{14-16}$ Although the obtained materials are of good quality, it is difficult to produce large-area (on the order of $0.1 \times 0.1 \mathrm{~m}^{2}$ ) thin films. As a possible means of overcoming this limitation, a polymer pyrolysis (PP) method for producing a graphite film from the solid phase by heating a polymer film at a high temperature has been extensively studied. ${ }^{17-19}$ The PP method was developed in 1986, and many studies have since been reported on the production of large-area graphite films and large graphite blocks. ${ }^{17,19}$ However, the thickness of

Received: May 13, 2019

Accepted: August 9, 2019

Published: August 9, 2019 
a

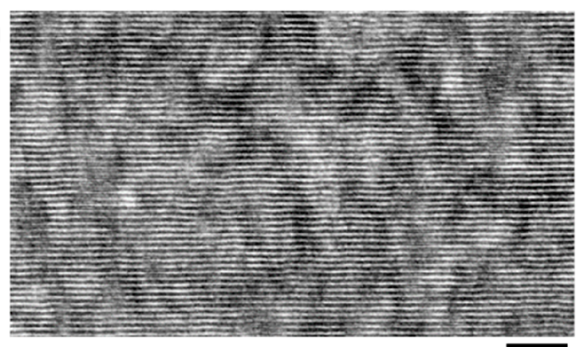

$3 \mathrm{~nm}$ b

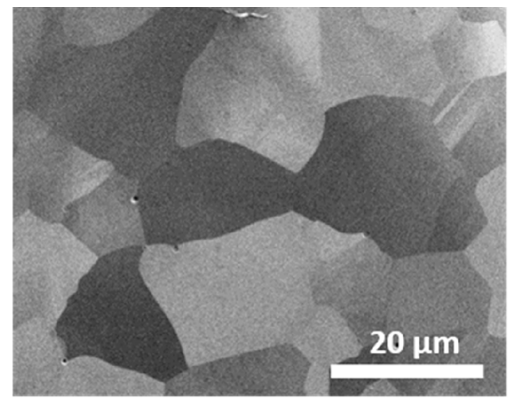

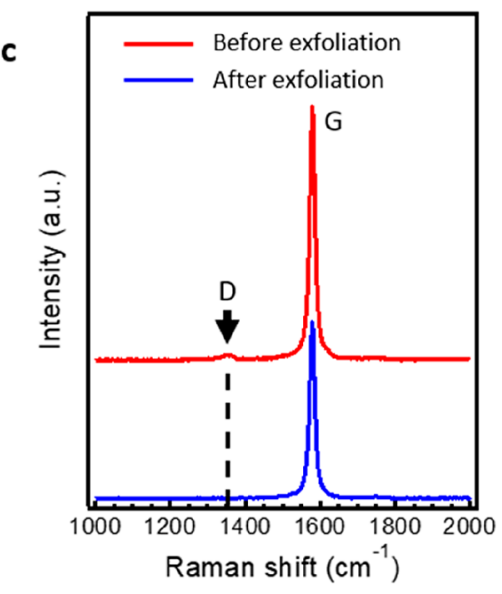

d

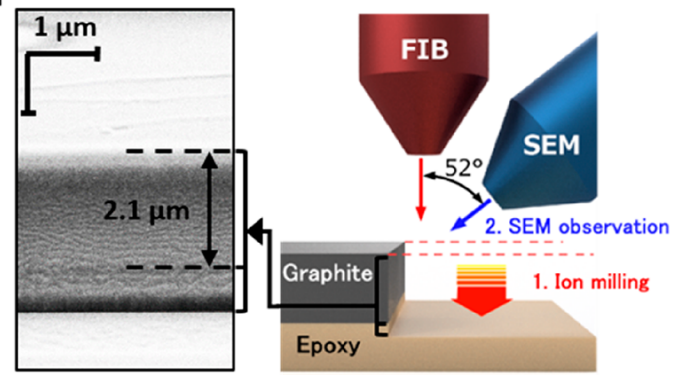

e
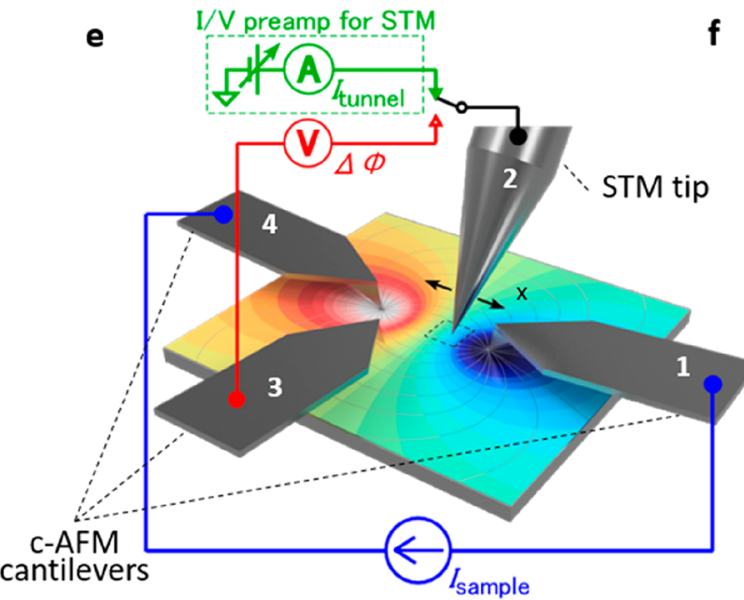

$\mathbf{f}$
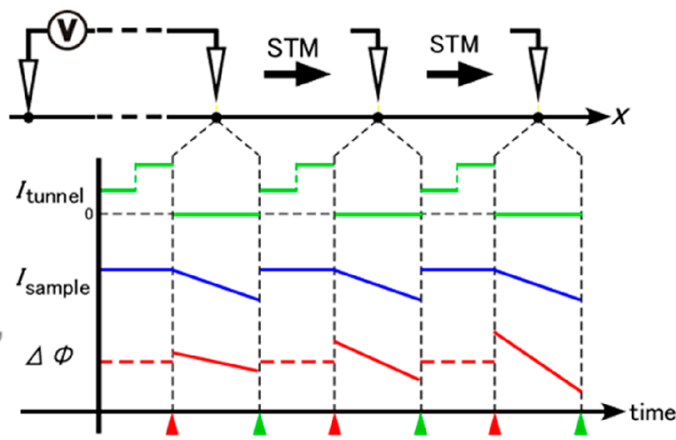

$\triangle$ STM feedback OFF \& potentiometry mode

$\triangle$ STM feedback ON \& current measurement mode

Figure 1. Sample conditions and schematic illustrations of measurements. (a) Cross-sectional TEM image of a graphite sample. (b) Secondary electron microscopy (SEM) image. (c) Raman spectra obtained before and after exfoliation. The D peak corresponds to defects. The G peak, which corresponds to other factors such as strain and the dopant used, is shown for comparison with the D peak in intensity. (d) FIB-SEM system used to produce a cross section of graphite film and a SEM image of the obtained sample. (e) Setup of the MP-STP measurement. (f) Measurement sequence.

the graphite thin films produced by the PP method has been limited to the range of $10-50 \mu \mathrm{m}$, and their electric and thermal conductivities were lower than those of Kish graphite and HOPG. ${ }^{20}$ With decreasing film thickness, the shrinkage and expansion of the graphite thin films during the growth process caused their breakage during the heating process. ${ }^{21}$ Therefore, the development of new techniques is highly desirable to produce thinner films.

Recently, the industrial growth of large-area high-quality graphite films with thicknesses from $\sim 0.5$ to $\sim 3 \mu$ m has been successfully realized by devising a handling technique for materials during the growth process, ${ }^{22}$ which provides a fundamental platform for examining and utilizing the intrinsic and ultimate characteristics of such materials. However, since the grain size of high-quality graphite films prepared by the PP method is on the order of micrometers, it is necessary to perform resistivity measurement on a similar scale, which has not yet been achieved. In general, the resistivity of graphite in the $a b$-plane has been measured using the conventional fourterminal method. ${ }^{14,23}$ When measurement is performed by reducing the distance of electrodes, methods such as ion beam etching and lithography are used to form a one-dimensional (1D) device structure. ${ }^{24,25}$ Therefore, it is difficult to clarify the effects of such physical and chemical processes on the characteristics of graphite. In addition, since graphite has large anisotropy between the resistivities in the $a b$-plane, $\rho_{a b}$, and along the $c$-axis, $\rho_{c}\left(\rho_{c} / \rho_{a b}=2500-5500^{26}\right.$ for pyrolytic graphite), the resistivity along the $c$-axis is an important factor. However, the resistivity in the $c$-axis direction has been measured by forming wiring on the surface of the graphite $a b$ - 
plane, ${ }^{26,27}$ and its direct measurement has not yet been realized because it is very difficult to prepare electrodes on the cross section of thin-film graphite by conventional methods such as lithography.

The four-probe method scanning tunneling microscopy (STM) was developed to measure the local electric conductivity of a sample. ${ }^{28}$ In this method, four probes are brought into contact with the sample. Current is allowed to flow between the two outer probes, and the voltage is measured by the two inner probes, similarly to the conventional four-terminal method. It is possible to perform local measurements without the complicated pretreatment of the sample. However, in general, the four probes come in contact with the sample.

On the other hand, scanning tunneling potentiometry (STP) is a method of measuring the local potential below a probe tip that does not come in contact with the sample. ${ }^{29,30}$ Current is allowed to flow between the two outer probes, and potentiometry is performed by using the inner STM probe. More accurate measurement becomes possible by adding a probe as a reference electrode (multiprobe STP: MP-STP). STM observation may also be performed simultaneously with the potentiometry measurement. However, when MP-STP is performed on a thin film such as graphite, the tip coming in contact with the film causes damage. In addition, the current path becomes three-dimensional (3D), and the direct measurement of the conductivity in the $c$-axis direction is required, which has not yet been achieved.

Here, we present the results obtained by MP-STP measurement in combination with the technique of atomic force microscopy (AFM). Local conductivity measurement was successfully performed on a $2.7 \mu \mathrm{m}$ thick graphite film grown by the PP method. The resistivity along the $c$-axis was directly obtained for the first time by MP-STP measurement over a cross section. The highest conductivity in the $a b$-plane, which is $\sim 1.3$ times that of single-crystal natural graphite (SCNG) and is considered to be an intrinsic and ultimate characteristics of multilayer graphite, was confirmed for the first time.

\section{RESULTS AND DISCUSSION}

Sample Preparation. Cracks and wrinkles forming in graphite films because of their shrinkage and expansion during the heating process have long been a technical problem. Generally, when the conventional industrial production method used for films thicker than $10 \mu \mathrm{m}$ is applied to the fabrication of films with thicknesses of $<3 \mu \mathrm{m}$, the films break and large-area films cannot be produced. Recently, we have improved the method of handling the polyimide (PI) membrane during the heating process, which is called the multipoint-support method and has enabled fabrication of high-quality large-area graphite thin films. Square graphite films with one side being $0.1 \mathrm{~m}$ and thicknesses from $\sim 0.5$ to $\sim 3 \mu \mathrm{m}$ were successfully grown (see the Methods section and ref 22 for details). As described in refs 17 and 22, although the quality of the sample deteriorates for a film with a thickness of about $10 \mu \mathrm{m}$ because of the insufficient graphitization inside the film, graphitization occurs throughout the entire film with a thickness of about $3 \mu \mathrm{m}$ or less. Therefore, in this experiment, measurement was performed by cleaving a sample of $\sim 2.7 \mu \mathrm{m}$ thickness (reduced to $\sim 2.1 \mu \mathrm{m}$ by cleaving) whose conductivity was highest in the $a b$-plane direction as confirmed by the macroscopic van der Pauw (VDP) ${ }^{31}$ method.
Figure 1a shows a transmission electron microscopy (TEM) image of the sample. It can be seen that the layered structure of graphite is well formed. Figure $1 \mathrm{~b}$ shows a scanning electron microscopy (SEM) image of the sample. The average grain size estimated by the channeling contrast method ${ }^{32,33}$ was $7.48 \mu \mathrm{m}$. Figure 1c shows Raman spectra obtained before and after exfoliation. The $\mathrm{D}$ peak, which corresponds to defects, disappeared after exfoliation, indicating the high quality of the sample at the level of Raman evaluation. No millimeterorder wrinkles, which are formed during the growth by the PP method, ${ }^{22}$ were detected as D signals in the Raman spectra.

In MP-STP measurements, unlike macroscale sheet resistance measurements, current is applied from the upper surface of the sample via an almost ideal point contact. Therefore, since the current path is an essential factor in the analysis, it is important to accurately measure the thickness of the sample. The graphite film was fixed onto a mica substrate by using an epoxy insulating adhesive. After the resistivity of the sample in the $a b$-plane was measured by MP-STP, which will be explained later, the sample was transferred to the chamber of a focused-ion-beam (FIB) SEM system (Helios NanoLab 600i, FEI Inc.) schematically shown in Figure 1d. Then, to expose a clean cross section, the part of the sample used for the $a b$-plane measurement was sputtered to a depth of $\sim 3 \mu \mathrm{m}$ perpendicular to the $a b$-plane by irradiating the surface with a focused $30 \mathrm{kV} \mathrm{Ga}{ }^{+}$ion beam in a vacuum chamber of $\sim 10^{-5} \mathrm{~Pa}$. A SEM image of the obtained cross section is shown in Figure 1d. Because the contrast in the graphite cross section and the epoxy part in the secondary electron image was clearly different, as shown in the SEM image in Figure $1 \mathrm{~d}$, the thickness of the graphite film was successfully determined to be $\sim 2.1 \mu \mathrm{m}$. The $\rho_{a b}$ of this sample which is cut to a $0.01 \times 0.01$ $\mathrm{m}^{2}$ square measured by the VDP method was $0.427 \mu \Omega \cdot \mathrm{m}$, the lowest resistivity within the $0.5-3 \mu \mathrm{m}$ thick films fabricated by the PP method. 22

MP-STP Measurement over the $a b$-Plane. Figure $1 \mathrm{e}$ shows the experimental setup of MP-STP. Because layered materials such as graphite are very fragile, it is necessary for the probe to come in contact with the sample without damaging its surface when measuring the intrinsic electric conductivity. Therefore, three conductive AFM (c-AFM) cantilevers ( $\mathrm{PtIr}_{5}$ coated, spring constant of $0.2 \mathrm{~N} / \mathrm{m}$, NANOSENSORS) were used as the contact probes. The contact between the cantilevers and the sample was confirmed by measuring the rapid increase in current. A constant current $\left(I_{\text {sample }}\right)$ was applied from probe 1 to probe 4 , and the reference potential was measured by using probe 3 . Because a soft contact by a cantilever tends to cause contact resistance fluctuations, a constant-current source was used instead of a constant-voltage source when applying a current to the sample. For STM and STP measurements, a probe made of electropolished tungsten (W) was used (probe 2). All the electric conduction measurements were performed in a UHV chamber $\left(\sim 10^{-7}\right.$ $\mathrm{Pa}$ ) at room temperature, and the location of the tip apex on the surface was determined by optical microscopy (working distance, $25 \mathrm{~mm}$; spatial resolution, $\sim 1 \mu \mathrm{m}$; Keyence Co., Ltd.) through the upper-side view port.

For the STM and STP measurements, a laboratory-built preamplifier was used to switch the circuit between the transimpedance amplifier mode (current measurement mode) and the potentiometer circuit mode (potentiometry mode) through a mechanical relay. Figure If shows a schematic of the STP measurement sequence. First, the preamplifier was set to 

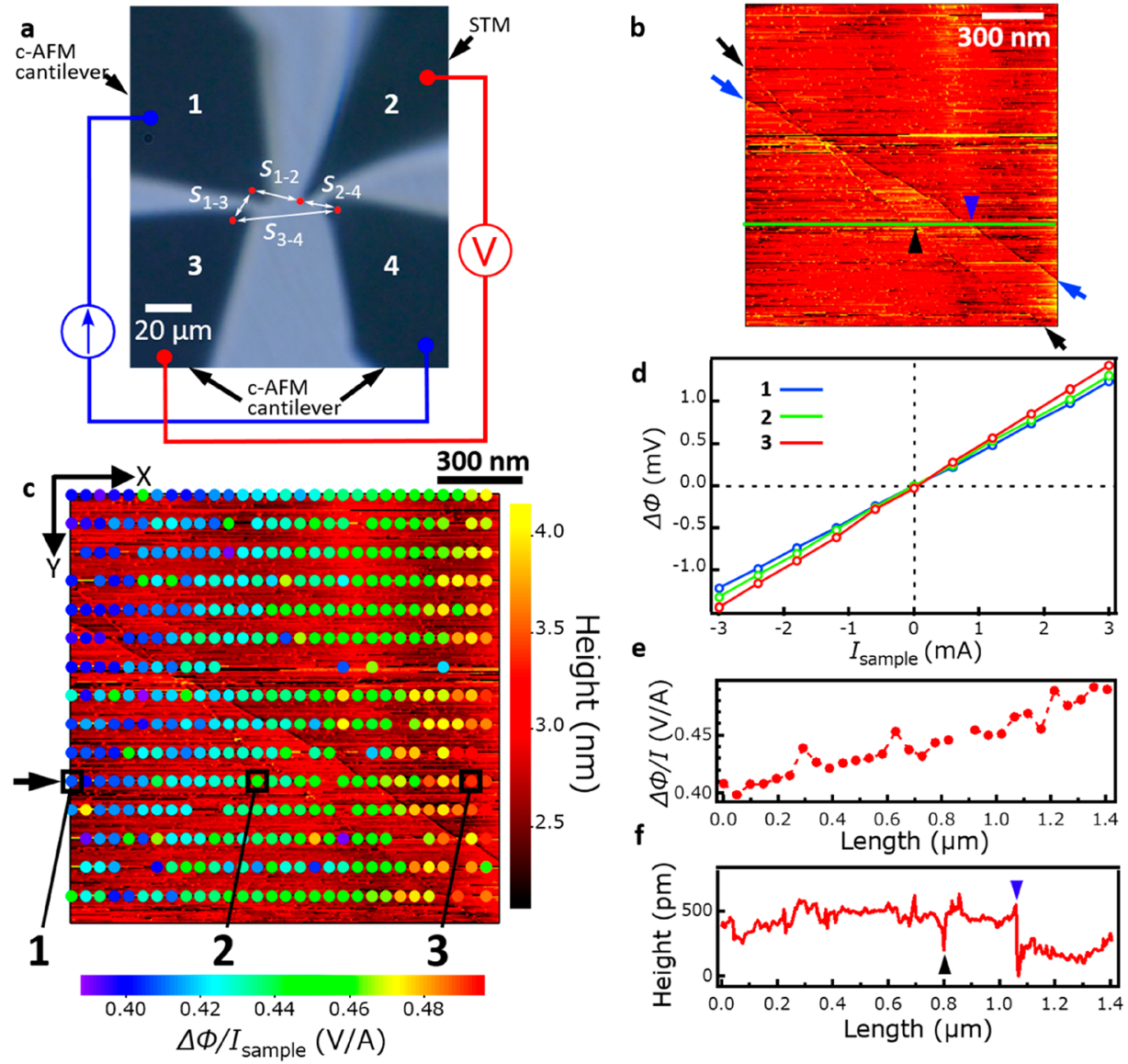

Figure 2. Results of MP-STM measurement in the ab-plane. (a) Optical microscopy image of the four probes during the measurement. (b) Topography image on which the grid measurement method was performed $\left(1.5 \times 1.5 \mu \mathrm{m}^{2}\right.$, tip bias $V_{\mathrm{t}}=20 \mathrm{mV}$, and set-point current $\left.I_{\mathrm{t}}=30 \mathrm{pA}\right)$. (c) $2 \mathrm{D}$ map of $\Delta \Phi / I_{\text {sample }}$ obtained over the surface in (b). The black arrow indicates the line corresponding to the green line in (b). (d) $\Delta \Phi-$ $I_{\text {sample }}$ plot acquired in the black rectangles in (c). (e) Line profile of $\Delta \Phi / I_{\text {sample }}$ along the green line in (b). (f) Line profile of the topographic image along the green line in (b).

the current measurement mode for STM imaging. Then, the scan was stopped at the measurement point in the image, and the tunnel resistance was decreased to about $10 \mathrm{M} \Omega$ orders by increasing the set-point current. Thereafter, the preamplifier was switched to the potentiometry mode, and the potential difference $\Delta \Phi$ between the area immediately below the STM tip and probe 3 was measured.

When the applied current $I_{\text {sample }}$ is swept linearly, $\Delta \Phi$ also changes linearly when the sample is metallic. Measurement errors, the fluctuation of the offset voltage due to such as the thermal electromotive force, can be eliminated by extracting the slope of $\Delta \Phi / I_{\text {sample }}$. In addition, nonlinearity was sometimes observed in the current-voltage characteristics between probe 1 and probe 4 because of the effect of adhered materials at the AFM cantilever-sample contact point. This is considered to have caused the resistance at the contact point to change at certain applied voltages. However, the nonlinear change in resistance at the contact points of probes 1 and 4 can be eliminated by carrying out potentiometry to measure the potential difference $\Delta \Phi$ between probes 2 and 3 with the fourtip probe method, while the position of probe 2 is changed. Namely, we can obtain the intrinsic resistivity of the sample by measuring $\Delta \Phi$ generated by the applied constant current $I_{\text {sample, }}$ which is not affected by the nonlinear characteristics of the contact points. In fact, a linear $\Delta \Phi-I_{\text {sample }}$ curve, which reflects the metallic characteristics of the sample, was obtained. ${ }^{34}$ By performing this process at each lattice point in the image during the STM scan, we obtained a 2D image of $\Delta \Phi / I_{\text {sample. }}$ Hereinafter, this measurement is called grid measurement.

The four-point probe method has been used for conductivity measurement in the past, and the validity of the analysis model has been confirmed by many measurements. ${ }^{35}$ In this experiment, the AFM technique was used to minimize the contact force between the tip and the sample to suppress the damage to the sample, but the mechanism is the same as that of the previous method. Therefore, analysis can be performed using the same model.

For a sample with isotropic conductivity, the resistivity $\rho$ can be represented as

$$
\rho=2 \pi \frac{\Delta \phi}{I_{\text {sample }}} \frac{1}{\left(\frac{1}{s_{\mathrm{is}, 1-2}}-\frac{1}{s_{\mathrm{is}, 2-4}}\right)-\left(\frac{1}{s_{\mathrm{ss}, 1-3}}-\frac{1}{s_{\mathrm{is}, 3-4}}\right)}
$$

where $s_{\text {iso, } n-m}$ is the distance between probe $n$ and probe $m$ (n, $m: 1,2,3,4)$ in the isotropic conduction model. To estimate the resistivity in the $a b$-plane, it is necessary to obtain an analytical formula by considering the conduction model (2D or $3 \mathrm{D})$. The large anisotropy between the resistivity in the $a b$ plane and that along the $c$-axis was converted into the model with an isotropic resistivity using the scaling law ${ }^{36,37}$ in 

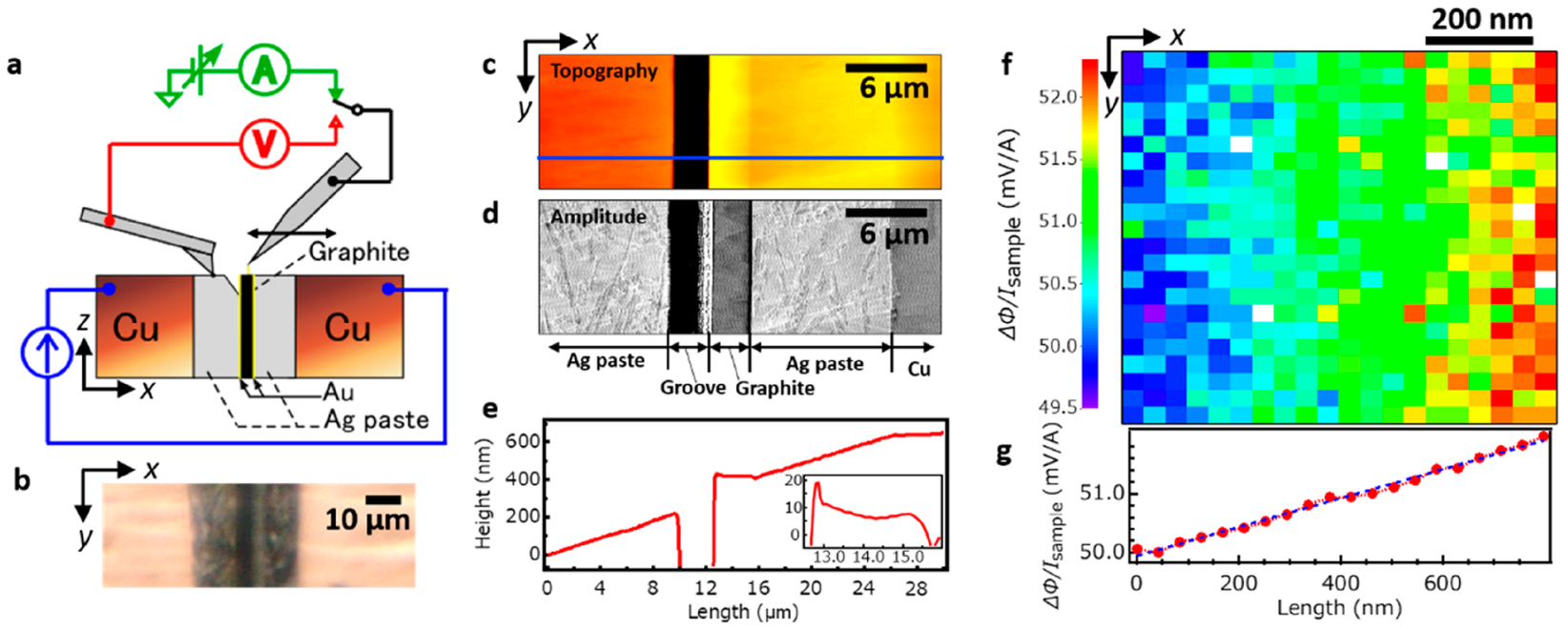

Figure 3. Results of cross-sectional MP-STM measurement. (a) Setup of the MP-STP measurement over the cross section. (b) Optical microscopy image of the upper side of the structure shown in (a). (c) Tapping AFM topographic image of the surface shown in (b). (d) Amplitude image (differential image) of the topographic image shown in (c). (e) Line profile along the blue line shown in the topography image in (c). (f) $2 \mathrm{D}$ distribution of $\Delta \Phi / I_{\text {sample }}$ obtained by the grid measurement over a scanning range of $800 \times 800 \mathrm{~nm}^{2}$. (g) Line profile of $\Delta \Phi / I_{\text {sample }}$ along the $x$ direction, in which $\Delta \Phi / I_{\text {sample }}$ was averaged in the $y$-direction.

$$
\begin{aligned}
& i_{\text {iso }}=i \sqrt{\frac{\rho_{i}}{\rho}} \quad(i=x, y, z) \\
& \rho=\sqrt[3]{\rho_{a b}{ }^{2} \rho_{c}}
\end{aligned}
$$

where $(x, y, z)$ and $\left(x_{\text {iso }}, y_{\text {iso }}, z_{\text {iso }}\right)$ represent the axes in the material with an anisotropic resistance and those in the converted space with an isotropic resistance, respectively. The $x$ - and $y$-axes are in the $a b$-plane, and the $z$-axis corresponds to the $c$-axis direction. For a typical ratio of the electric conductivity of graphite $\left(\rho_{c} / \rho_{a b}=\sim 5000^{26}\right)$, when the length in the $a b$-plane and that along the $c$-axis are multiplied by 0.24 and 17 , respectively, the model can be transformed into a rectangular model with an isotropic conductivity. Whether the conduction model is $2 \mathrm{D}$ or $3 \mathrm{D}$ is determined by the ratio of $L^{\prime}{ }_{1-4}$ (distance between probe 1 and probe 4 after scaling) to $t^{\prime}$ (the thickness of graphite after scaling); the model becomes $3 \mathrm{D}$ when $t^{\prime} / L_{1-4}^{\prime}>0.75 .^{38,39}$ In this experiment, by use of $L_{1-4}$ $=23 \mu \mathrm{m}$ and $t=2.1 \mu \mathrm{m}$, the ratio is $t^{\prime} / L^{\prime}{ }_{1-4}=8.82\left(L^{\prime}{ }_{1-4}=5\right.$ $\left.\mu \mathrm{m}, t^{\prime}=44.1 \mu \mathrm{m}\right)$, and a 3D model was employed. Finally, the analytic equation of the $3 \mathrm{D}$ conduction model considering the electric conductivity anisotropy between $\rho_{a b}$ and $\rho_{c}$ is expressed as

$$
\rho_{a b}=\left(2 \pi \frac{\Delta \phi}{I_{\text {sample }}} \frac{1}{\left(\frac{1}{s_{1-2}}-\frac{1}{s_{2-4}}\right)-\left(\frac{1}{s_{1-3}}-\frac{1}{s_{3-4}}\right)}\right)^{2} \frac{1}{\rho_{c}}
$$

where $s_{n-m}$ is the actual distance between probe $\mathrm{n}$ and probe $m$ $(n, m: 1,2,3,4)$. By applying the coordinate transformation of eq 2 to the interprobe distance $s_{\text {iso }, n-m}$ in eq 1 , and substituting $\rho$ of eq 2 into eq 1 , we obtain eq 3 , which represents a model of the sample with anisotropic conductivity. Experiments were performed to obtain $\rho_{a b}$ by measuring $\rho_{c}$ and the other parameters.

Figure 2a shows an optical microscopy image of the four probes during the measurement. As the current $I_{\text {sample }}$ flows from probe 1 to probe $4, \Delta \Phi$ between probe 2 and probe 3 was determined by a grid measurement. The scanning direction $x$ of probe 1 was adjusted to be along the direction of $I_{\text {sample }}$. The position of each probe was determined from the optical microscopy image considering the configuration of the cantilever, and the distances between the probes were estimated to be $s_{1-2}=\sim 9 \mu \mathrm{m}, s_{2-4}=\sim 27 \mu \mathrm{m}, s_{1-3}=\sim 16$ $\mu \mathrm{m}$, and $s_{2-4}=\sim 9 \mu \mathrm{m}$.

Figure $2 \mathrm{~b}$ shows a topography image obtained over the area where the grid measurement was performed $\left(1.5 \times 1.5 \mu \mathrm{m}^{2}\right.$, tip bias $V_{\mathrm{t}}=20 \mathrm{mV}$, and set-point current $I_{\mathrm{t}}=30 \mathrm{pA}$ ), and the values of $\Delta \Phi / I_{\text {sample }}$ are shown in Figure $2 \mathrm{c}$ in a color scale. Although measurements at some points are missing in Figure $2 \mathrm{c}$ because we could not obtain the linear $I-V$ curves at those points probably because of the fluctuations in the tunnel junction, atomic layer steps and grain boundaries were observed as indicated by the blue and black arrows, respectively. ${ }^{40}$ As shown in Figure $2 \mathrm{c}, \Delta \Phi / I_{\text {sample }}$ increased from left to right. The line indicated by the black arrow in Figure $2 c$ shows the results obtained along the green line in Figure $2 \mathrm{~b}$, and the $\Delta \Phi-I_{\text {sample }}$ plot acquired in the black rectangles ( 1 to 3 ) is shown in Figure 2 d. Each curve shows a linear characteristic, indicating that the sample is metallic, and the slopes of the curves are clearly different. The distribution of $\Delta \Phi / I_{\text {sample }}$ will later be discussed in comparison with the calculated results.

The line profiles of $\Delta \Phi / I_{\text {sample }}$ and the topographic image along the green line in Figure $2 \mathrm{~b}$ are shown in Figures $2 \mathrm{e}$ and $2 \mathrm{f}$, respectively. The $\Delta \Phi / I_{\text {sample }}$ plot is not sensitive to the surface structures; namely, there is no correspondence between the $\Delta \Phi / I_{\text {sample }}$ plot shown in Figure $2 \mathrm{e}$ and the structures of the atomic layer step and the grain boundary indicated by the blue and black triangles in Figures $2 \mathrm{~b}$ and $2 \mathrm{f}$. Namely, no voltage drop was observed at the grain boundaries.

Cross-Sectional STP Experiment and Analysis. To experimentally obtain $\rho_{c}$, we performed MP-STP measurement over a cross section, as schematically shown in Figure 3a (see the Methods section for details). Figure $3 b$ shows an optical microscopy image of the upper side of the structure shown in Figure 3a. While applying a constant current through the $\mathrm{Cu}$ plates, the STM probe was made to approach the graphite 

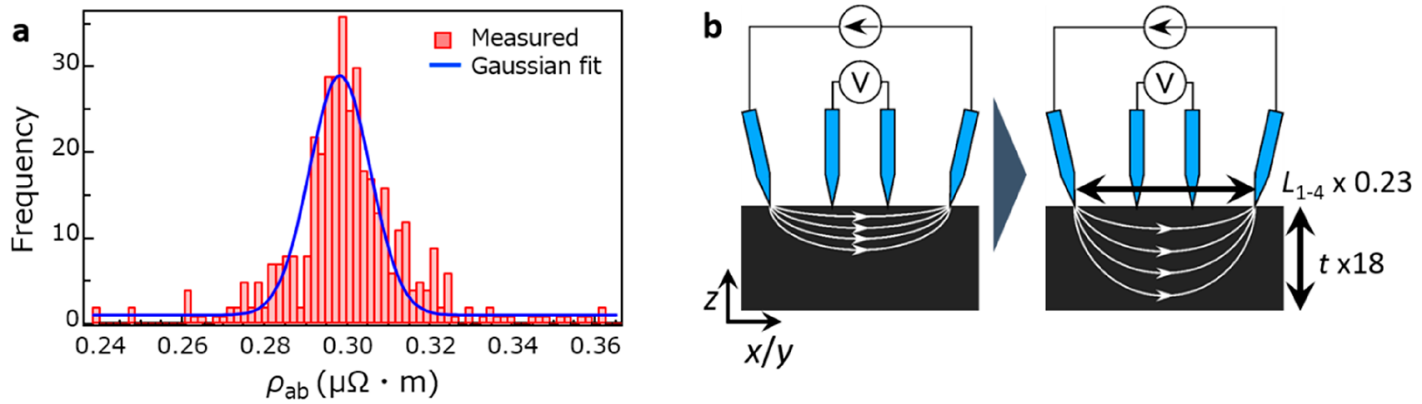

C
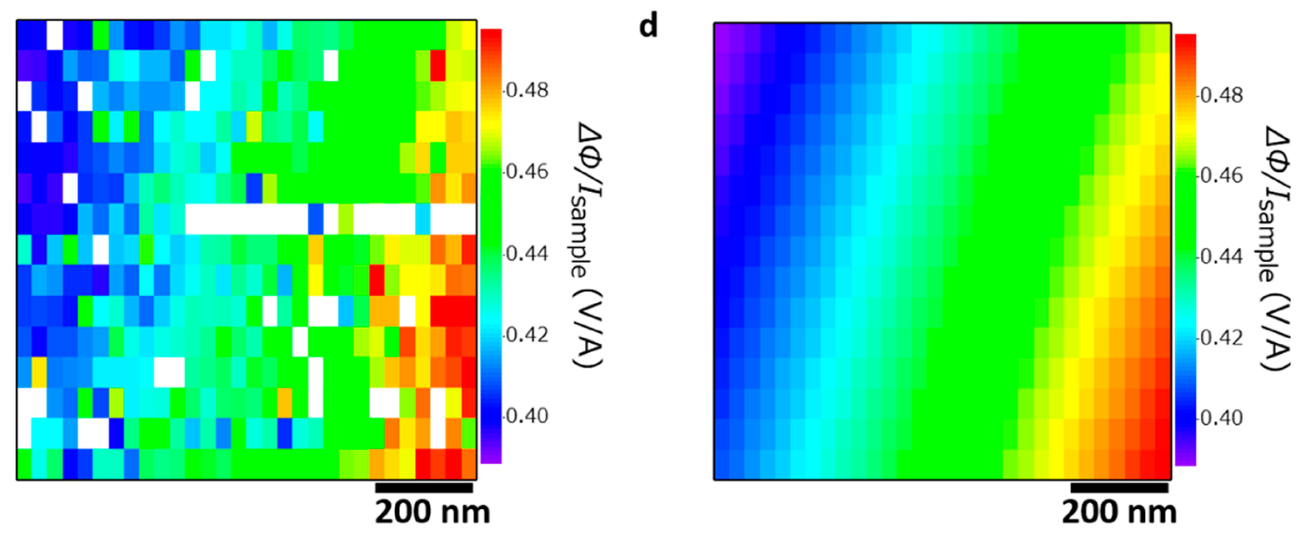

Figure 4. Experimental and calculated results $\rho_{a b}$. (a) Histogram of $\rho_{a b}$ calculated using $\Delta \Phi / I_{\text {sample }}$ obtained at each grid point in Figure 2c. (b) Scaling model from the original structure with high anisotropic resistivity (left) to the structure with isotropic resistivity. (c) $2 \mathrm{D}$ distribution of $\Delta \Phi / I_{\text {sample }}$ shown in Figure 2c. (d) $\Delta \Phi / I_{\text {sample }}$ obtained by calculation.

cross section under observation with an optical microscope, and $\Delta \Phi$ between the STM probe and the cantilever located in the Ag epoxy part was measured by STP.

Figure $3 c$ shows a topographic image of the surface shown in Figure $3 \mathrm{~b}$ obtained before the STP measurement by tapping AFM in the constant-amplitude mode. Figure $3 \mathrm{~d}$ shows the amplitude image (differential image) of the topographic image, in which each edge region is clearly determined. The deep groove between the graphite and the silver epoxy on the left side is clearly observed. Figure $3 e$ shows the line profile along the blue line shown in the topography image in Figure 3c. It was confirmed that the graphite part had a very flat cross section with a roughness of $<3 \mathrm{~nm}$. The slope observed on both sides is considered to have been formed during the cutting process.

Figure $3 \mathrm{f}$ shows the $2 \mathrm{D}$ distribution of $\Delta \Phi / I_{\text {sample }}$ obtained by grid measurement over a scanning range of $800 \times 800 \mathrm{~nm}^{2}$. The scan direction $x$ was parallel to the current direction. The feedback conditions were $V_{\mathrm{t}}=-3 \mathrm{mV}$ and $I_{\mathrm{t}}=10 \mathrm{pA}$. The topography image was acquired simultaneously with the STP measurement, and the cross-sectional surface was confirmed to be very flat (roughness $\mathrm{p}-\mathrm{p}<3 \mathrm{~nm}) . \Delta \Phi / I_{\text {sample }}$ was measured on each grid point by measuring $\Delta \Phi$ while changing $I_{\text {sample }}$ stepwise between -9 and $+9 \mathrm{~mA}$. In this experiment, no nonlinearity was observed in the current-voltage characteristics at the contact point because of the formation of a wide contact area; therefore, $\Delta \Phi$ was obtained from the difference between the two points $(-9$ and $+9 \mathrm{~mA}) . \Delta \Phi / I_{\text {sample }}$ increases from left to right. The values averaged in the $y$-direction are shown in Figure 3g. The linearity was confirmed, from which the slope of $\Delta \Phi /\left(I_{\text {sample }} d\right)$ was obtained to be $2.45 \pm 0.05 \mathrm{~V}$ $\mathrm{A}^{-1} \mathrm{~m}^{-1}$, where $d$ is the length of the measured area along the $x$-direction. Therefore, $\rho_{c}$ can be obtained from the simple 1D conduction model as

$$
\rho_{c}=\frac{S \Delta \phi}{I_{\text {sample }} d}
$$

where $S$, the area over which the graphite sample was measured by optical microscopy, was estimated to be $0.703 \mathrm{~mm}^{2}$. As shown in Figure 3a, a constant current was applied between the electrodes attached to both sides of the sample, and the voltage drop between them was measured by the four-probe method. Because the size of the $a b$-plane of the sample used is $1 \mathrm{~mm} \times 0.7 \mathrm{~mm}$, and the bulk part is very large compared with the edge area, the current that flowed on the bulk side is considered to be approximately equal to the applied constant current $I_{\text {sample }}$. As a result, $\rho_{c}$ was determined to be $1.72 \pm 0.04$ $\mathrm{m} \Omega \cdot \mathrm{m}$.

Next, $\rho_{a b}$ was estimated from the data obtained from eq 3 and the value of $\rho_{c}$. Figure $4 \mathrm{a}$ shows a histogram of $\rho_{a b}$ calculated using the value of $\Delta \Phi / I_{\text {sample }}$ obtained at each grid point in Figure $2 \mathrm{c}$ by measuring the relationship shown in Figure $2 \mathrm{~d}$. From the peak value of the Gaussian fitting and its half-width at half-maximum, $\rho_{a b}$ was estimated to be $0.30 \pm$ $0.01 \mu \Omega \cdot \mathrm{m}$. From these results, $\rho_{c} / \rho_{a b}$ was found to be $\sim 5.7 \times$ $10^{3}$. With this value, as shown in Figure $4 \mathrm{~b}$, if the length in the $x$ - and $y$-directions is compressed 0.23 times and the length along the $z$-axis is stretched 18 times, transformation into an isotropic resistivity structure is realized. The scaling law applied to the isotropic conduction model was found to be $t^{\prime}$ / $L^{\prime}{ }_{1-4}=7.09\left(t^{\prime}=37.5 \mu \mathrm{m}, L^{\prime}{ }_{1-4}=\sim 5.3 \mu \mathrm{m}\right)$. Because $t^{\prime} / L^{\prime}{ }_{1-4}$ $>0.75$, it was confirmed that the application of the $3 \mathrm{D}$ model was reasonable.

Finally, the $2 \mathrm{D}$ distribution of $\Delta \Phi / I_{\text {sample }}$ was calculated from the values of $\rho_{a b}$ and $\rho_{c}$. For comparison, the $\Delta \Phi / I_{\text {sample }}$ map in Figure $2 c$ is shown in Figure $4 c$. Figure $4 d$ shows the map of $\Delta \Phi / I_{\text {sample }}$ obtained by calculation using eq 3 . Although the experimental values fluctuate because of the 
a

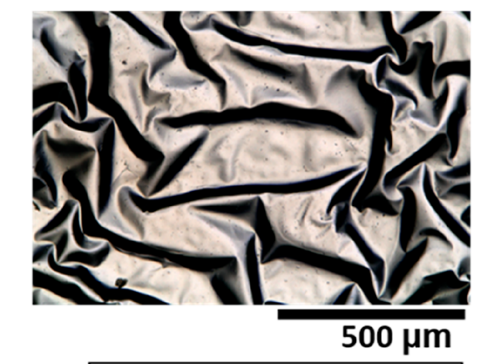

$\mathbf{f}$
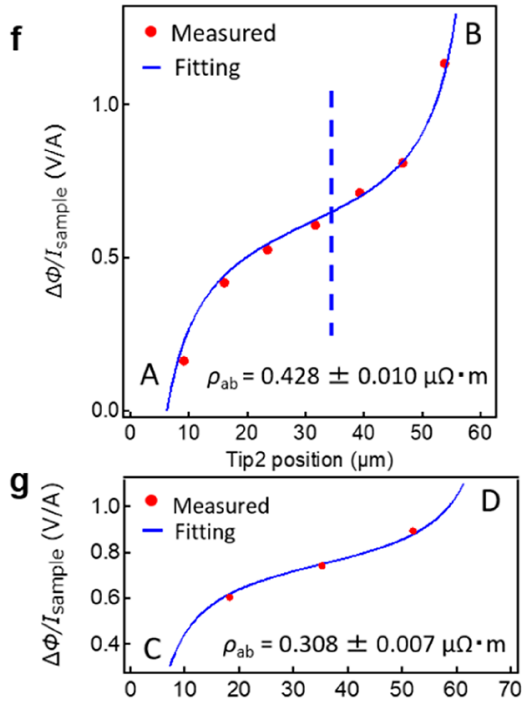

b

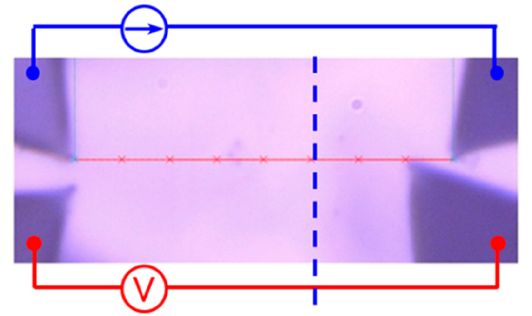

C

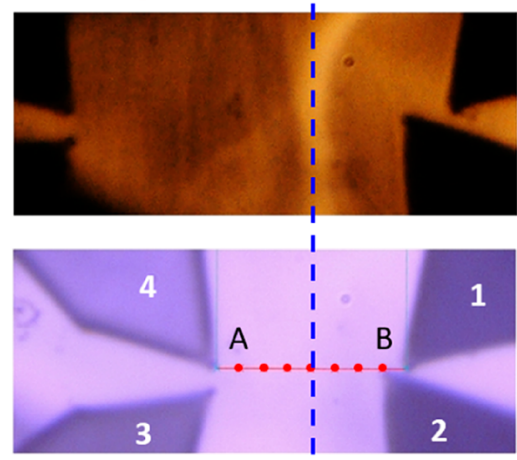

e

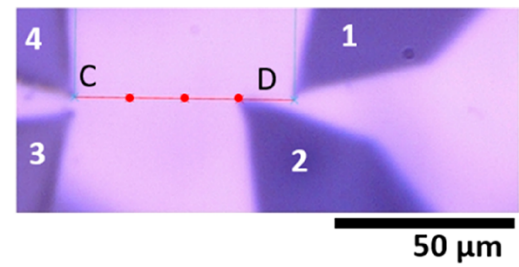

Figure 5. Effect of wrinkles on the resistance of $\rho_{a b}$. (a) Optical image of the wrinkles of the sample. (b) Optical image of the measured area with a wrinkle. (c) Optical image of the same area in (b) with a different light angle to make the wrinkle more visible. (d) Measurement area including a wrinkle. (e) Measurement area without wrinkle. (f) $\Delta \Phi / I_{\text {sample }}$ as a function of the position of tip 2. A and B correspond to those in (d). (g) $\Delta \Phi /$ $I_{\text {sample }}$ as a function of the position of tip 2. C and D correspond to those in (e). Blue solid lines in (f) and (g) show the fitting curves obtained by using $\rho_{a b}$ as a parameter while retaining $\rho_{c}$ constant.

noise and the instability of the probe state, as shown in the histogram in Figure 4a, it was confirmed that the observed characteristics are consistent with the calculated values.

The $\rho_{a b}$ measured by the MP-STM method was estimated to be $0.30 \pm 0.01 \mu \Omega \cdot \mathrm{m}$, which was about $30 \%$ lower than that $(0.427 \mu \Omega \cdot \mathrm{m})$ obtained by the macroscale VDP method. This difference may be due to the fact that the measurement by the VDP method being performed in a region including grain boundaries. We made a simple estimation of their effects on the conductance.

In the case of a metallic material, the resistivity is expressed by Matthiesen's rule as

$$
\rho=\rho_{T}+\rho_{\text {def }}+\rho_{\text {imp }}
$$

where $\rho_{T}$ is the temperature-dependent term and the main factor, which originates from the scattering by phonons. $\rho_{\text {imp }}$ is the term representing scattering due to impurities, which is considered to be independent of temperature. $\rho_{\text {def }}$ is the term related to defects. At room temperature, $\rho_{T}$ is expected to be the dominant term, which is included in both MP-STP and VDP measurements. Therefore, assuming that the impurity level is low because of the controlled sample preparation environment, and thus the term $\rho_{\text {imp }}$ is negligible, the difference between the results of MP-STP and VDP measurements is considered to be due to $\rho_{\text {def }}$.

We discuss this result by converting the measurement conditions of a $0.01 \times 0.01 \mathrm{~m}^{2}$ sheet of $2 \mu \mathrm{m}$ thickness to a simple $1 \mathrm{D}$ resistance model. By use of an average grain size of
$7.48 \mu \mathrm{m}$, the number of grain boundaries contained in each measurement area is roughly estimated to be $\sim 2$ by MP-STP and $\sim 1337$ by VDP. From these results and the resistivity $\rho_{a b}$, the $1 \mathrm{D}$ resistance of the entire sheet is estimated to be $\sim 0.15$ $\Omega$ by MP-STP $\left(=R_{1 \mathrm{D}, \mathrm{MPSTP}}\right)$ and $\sim 0.21 \Omega$ by VDP (= $\left.R_{1 \mathrm{D}, \mathrm{VDP}}\right)$. Assuming that the difference of $\sim 0.06 \Omega(0.21-0.15$ $\Omega$ ) was only due to the difference in the number of grain boundaries contained in the measurement area, the resistance is $\sim 4.5 \times 10^{-5} \Omega(0.06 / 1335 \Omega)$ per grain boundary. Using the grain boundary width of $10 \mathrm{~nm}^{41}$ and the sample crosssectional size $(0.01 \mathrm{~m} \times 2 \mu \mathrm{m})$, we estimated the resistivity of a grain boundary to be $9.0 \times 10^{-5} \Omega \cdot \mathrm{m}$, which is lower than that in graphene reported previously $\left(10^{-3}-10^{-1} \Omega \cdot \mathrm{m}\right){ }^{41,42}$ The voltage drop at the grain boundaries is calculated to be $\sim 675$ $\mu \mathrm{V}$ using the current density of $\sim 7.5 \times 10^{-4} \mathrm{~A} / \mu \mathrm{m}^{2}$ in the MPSTP measurement and the resistance of $\sim 4.5 \times 10^{-5} \Omega[7.5 \times$ $\left.10^{-4} \mathrm{~A} / \mu \mathrm{m}^{2} \times(0.01 \mathrm{~m} \times 2 \mu \mathrm{m}) \times\left(4.5 \times 10^{-5} \Omega\right)\right]$ per a grain boundary. However, as shown in Figure 2e, the cross section microscopically obtained is almost linear, and the voltage drop observed in this experiment is less than the resolution of the system $(\sim 10 \mu \mathrm{V})$.

The observed reduction in resistivity may be attributed to the effects originated from multilayer graphene. The effect of, for example, grain boundaries on the conductivity, which is very high in graphene, ${ }^{41-44}$ is expected to be reduced in multilayer graphene because more conductive paths are formed in a multilayer structure because of the different locations of domains in the layers along the $c$-axis direction, parallel to the 
2D sheet. In such a case, the effect of grain boundaries is reduced because current flows avoid the boundaries.

Therefore, what causes the difference between the values obtained by VDP and MP-STP methods? One possibility is the effect of the wrinkles shown in Figure 5a, which formed on the sample during heat treatment. Here, the sample was illuminated from right above, and the wrinkles in the direction of the shadow appeared widespread and black. Although it has been considered that the wrinkles formed are smooth, as observed in Figure $5 b$, and do not affect the resistance, to clarify its effect in more detail, the area including a wrinkle was measured by the four-point probe method.

Figure $5 \mathrm{c}$ shows an image of the same area shown in Figure $5 \mathrm{~b}$, in which the irradiation angle of light was changed to make the wrinkle appear. Figures $5 \mathrm{f}$ and $5 \mathrm{~g}$ show the results of measurement across a wrinkle as shown in Figure $5 \mathrm{~d}$ and in the area without wrinkles shown in Figure 5e, respectively. Fitting was performed using eq 3 , where $\rho_{c}$ was assumed to be constant and $\rho_{a b}$ was used as a fitting parameter. In Figure 5f, the obtained $\rho_{a b}(=0.428 \pm 0.01 \mu \Omega \cdot \mathrm{m})$ was almost the same as that $(0.427 \mu \Omega \cdot \mathrm{m})$ obtained by the VDP method, ${ }^{22}$ whereas the $\rho_{a b}(=0.31 \pm 0.01 \mu \Omega \cdot \mathrm{m})$ obtained for the area shown in Figure $5 \mathrm{~g}$ was in good agreement with that obtained for the area shown in Figure $2(0.30 \pm 0.01 \mu \Omega \cdot \mathrm{m})$. That is the value obtained by VDP is affected by the smooth wrinkles. The slight deviation difference of the fitting curve from the experimental values between the left and right of the dotted line in Figure $5 \mathrm{f}$ may be due to the fact that the change in resistance is localized near the area surrounding the wrinkle.

SCNG is the graphite with the highest known conductivity, and although it is the result of measurement at the conventional macroscopic (millimeters) scale, we have used it because of its ideal structure without grain boundaries or wrinkles for comparison. However, the conductivity of SCNG is considered to be determined by the impurities contained during the crystal growth in addition to the effect of phonon vibration. In ref 47, the residual resistance (resistance components other than thermal phonons) of SCNG is shown to be $0.1 \mu \Omega \cdot \mathrm{m}$ or less. This value is in good agreement with $0.08 \mu \Omega \cdot \mathrm{m}$, which is the difference between the current measurement result $(0.30 \mu \Omega \cdot \mathrm{m})$ and that of SCNG $(0.38 \mu \Omega$. $\mathrm{m})$.

A more detailed examination of the area with wrinkles should be further studied, but the elimination of wrinkles is expected to provide high conductivity comparable to that in the ideal area. A method of eliminating wrinkles is currently underway. In addition, when forming thinner films by, for example, the chemical etching of multilayer graphite, it becomes increasingly difficult to evaluate the characteristics in the $c$-axis direction, and the method developed in this study is expected to play a key role. Analysis of dynamics by timeresolved measurement may give important information. ${ }^{45,46}$

\section{CONCLUSION}

We performed measurements by MP-STP on a high-quality 2.7 $\mu \mathrm{m}$ thick graphite film with a large area fabricated by the PP method. In addition to the measurement of $\rho_{a b}$ over an $a b$ plane without wrinkles, MP-STP measurement over a cross section perpendicular to the $a b$-plane to acquire $\rho_{c}$ along the $c$ axis was successfully achieved for the first time. The $\rho_{a b}$, which was measured to be $0.427 \mu \Omega \cdot \mathrm{m}$ by the VDP method, was found to be $0.30 \pm 0.01 \mu \Omega \cdot \mathrm{m}$ by MP-STP; that is, it was extremely lower than the previously reported lowest value of
$0.38 \mu \Omega \cdot \mathrm{m}$ for SCNG. ${ }^{47}$ Although it is the result of fourterminal measurement at the conventional macroscopic (millimeters) scale, SCNG is the graphite with the highest known conductivity because of its ideal structure without grain boundaries or wrinkles. In fact, the residual resistance of SCNG, $0.1 \mu \Omega \cdot \mathrm{m}$ or less, is in good agreement with $0.08 \mu \Omega$. $\mathrm{m}$, which is the difference between the current measurement result $(0.30 \mu \Omega \cdot \mathrm{m})$ and that of $\operatorname{SCNG}(0.38 \mu \Omega \cdot \mathrm{m})$. Therefore, the highest conductivity in the ab-plane, $\sim 1.3$ times that of SCNG, may be the intrinsic and ultimate characteristic of multilayer graphite. The advantage of multilayer graphene, which reduces the effect of grain boundaries on conductance, was successfully confirmed. Therefore, when the density of wrinkles is decreased by improving the manufacturing conditions, it becomes possible to industrially produce large-area highest-quality graphite, which has never been produced. This is a major progress toward the realization of its future applications.

\section{METHODS}

Sample Synthesis. Sample graphite films were prepared as follows. Highly orientated PDMA/ODA (PDMA: 3,3',4,4'-biphenyltetracarboxylic dianhydride; ODA: 4,4'-diaminodiphenyl ether) type PI films were used as raw materials. First, carbonization was performed by heating the films at $\sim 1000{ }^{\circ} \mathrm{C}$ in a nitrogen flow and then hollowed by using a graphite heater with the temperature increased to $3300{ }^{\circ} \mathrm{C}$ in an argon flow (heating rate: $20{ }^{\circ} \mathrm{C} / \mathrm{min}$; retention time at $\left.3300{ }^{\circ} \mathrm{C}: 5 \mathrm{~min}\right) .^{21,22}$

Sample Preparation for Cross-Sectional STP. First, after the vapor deposition of $200 \mathrm{~nm}$ thick gold $(\mathrm{Au})$ along the $a b$-plane on both sides of a graphite sample, the sample was cut into a rectangular shape $\left(\sim 0.5 \times 1.5 \mathrm{~mm}^{2}\right)$, and a $0.3 \mathrm{~mm}$ thick copper $(\mathrm{Cu})$ plate of approximately the same size was adhered to each $200 \mathrm{~nm}$ thick $\mathrm{Au}$ layer, using a conductive silver $(\mathrm{Ag})$ epoxy paste (resistivity $\leq 4 \mu \Omega$. $\mathrm{m}$ ). At the same time, an insulating epoxy paste was applied to the edge of the graphite to prevent a short circuit between the $\mathrm{Cu}$ plates on both sides and the graphite edges and to accurately maintain the thickness of the graphite sample contributing to the measurement. Subsequently, the sample was cut roughly with a diamond cutter, and then a flat cross-sectional surface was formed by Ar ion sputtering ( 6 $\mathrm{kV}$ acceleration voltage, $10^{-5} \mathrm{~Pa}$ ) by using a cross-sectional polisher (IM 4000 PLUS, Hitachi High-Technologies Corp.). A deep groove was observed between the graphite and the silver epoxy on the left side, as shown in Figure $3 a-e$, which was formed because of the shrinkage of the silver epoxy caused by the heat generated during the sputtering with the Ar beam.

\section{ASSOCIATED CONTENT}

Supporting Information

The Supporting Information is available free of charge on the ACS Publications website at DOI: 10.1021/acsaelm.9b00298.

Electron backscattered diffraction pattern (EBSD) image, SEM image (PDF)

\section{AUTHOR INFORMATION}

\section{Corresponding Author}

*E-mail: hidemi@ims.tsukuba.ac.jp.

ORCID

Hidemi Shigekawa: 0000-0001-9550-5148

\section{Author Contributions}

H.M., T.B., and A.T. performed the experiments. M.M. and Y.K. developed the PP method to grow high quality graphite sample films. M.Y. evaluated the sample quality by Raman spectroscopy and the EBSD method. S.Y. and O.T. provided 
technical advice. H.S. organized and supervised the project and edited the paper with H.M. and the other authors.

\section{Notes}

The authors declare no competing financial interest.

\section{ACKNOWLEDGMENTS}

H.S. acknowledges the financial support of a Grant-in-Aid for Scientific Research (17H06088) from Japan Society for the Promotion of Science. This work was supported in part by University of Tsukuba Nanofabrication Platform and the AIST Nano-Processing Facility in the "Nanotechnology Platform Project" sponsored by the Ministry of Education, Culture, Sports, Science and Technology (MEXT), Japan.

\section{REFERENCES}

(1) Novoselov, K. S.; Geim, A. K.; Morozov, S. V.; Jiang, D.; Zhang, Y.; Dubonos, S. V.; Grigorieva, I. V.; Firsov, A. A. Electric Field Effect in Atomically Thin Carbon Films. Science 2004, 306, 666-669.

(2) Wei, H.; Xiao, J.; Yang, Y.; Lv, S.; Shi, J.; Xu, X.; Dong, J.; Luo, Y.; Li, D.; Meng, Q. Free-Standing Flexible Carbon Electrode for Highly Efficient Hole-Conductor-Free Perovskite Solar Cells. Carbon 2015, 93, 861-868.

(3) Xu, Y.; Yu, H.; Wang, C.; Cao, J.; Chen, Y.; Ma, Z.; You, Y.; Wan, J.; Fang, X.; Chen, X. Multilayer Graphene with Chemical Modification as Transparent Conducting Electrodes in Organic LightEmitting Diode. Nanoscale Res. Lett. 2017, 12, 254.

(4) Anagnostopoulos, G.; Pappas, P. N.; Li, Z.; Kinloch, I. A.; Young, R. J.; Novoselov, K. S.; Lu, C. Y.; Pugno, N.; Parthenios, J.; Galiotis, C.; Papagelis, K. Mechanical Stability of Flexible GrapheneBased Displays. ACS Appl. Mater. Interfaces 2016, 8, 22605-22614.

(5) Kim, J. Y.; Cho, N. S.; Cho, S.; Kim, K.; Cheon, S.; Kim, K.; Kang, S. Y.; Cho, S. M.; Lee, J. I.; Oh, J.; Kim, Y. H.; Ryu, H.; Hwang, C. S.; Kim, S.; Ah, C. S.; Kim, T. Y. Graphene Electrode Enabling Electrochromic Approaches for Daylight-Dimming Applications. Sci. Rep. 2018, 8, 3944.

(6) Bae, S.; Kim, H.; Lee, Y.; Xu, X.; Park, J. S.; Zheng, Y.; Balakrishnan, J.; Lei, T.; Ri Kim, H.; Song, Y. Il; Kim, Y. J.; Kim, K. S.; Özyilmaz, B.; Ahn, J. H.; Hong, B. H.; Iijima, S. Roll-to-Roll Production of 30-Inch Graphene Films for Transparent Electrodes. Nat. Nanotechnol. 2010, 5, 574-578.

(7) Yazami, R.; Touzain, P. A Reversible Graphite-Lithium Negative Electrode for Electrochemical Generators. J. Power Sources 1983, 9, $365-371$.

(8) Blomquist, N.; Wells, T.; Andres, B.; Bäckström, J.; Forsberg, S.; Olin, H. Metal-Free Supercapacitor with Aqueous Electrolyte and Low-Cost Carbon Materials. Sci. Rep. 2017, 7, 39836.

(9) Yu, A.; Roes, I.; Davies, A.; Chen, Z. Ultrathin, Transparent, and Flexible Graphene Films for Supercapacitor Application. Appl. Phys. Lett. 2010, 96, 253105.

(10) Hannay, N. B.; Geballe, T. H.; Matthias, B. T.; Andres, K.; Schmidt, P.; MacNair, D. Superconductivity in Graphitic Compounds. Phys. Rev. Lett. 1965, 14, 225-226.

(11) Belash, I.; Zharikov, O.; Palnichenko, A. Superconductivity of GIC with Li, Na and K. Synth. Met. 1989, 34, 455-460.

(12) Larkins, G.; Vlasov, Y.; Holland, K. Evidence of Superconductivity in Doped Graphite and Graphene. Supercond. Sci. Technol. 2016, 29, 015015.

(13) Cui, X.; Lee, G. H.; Kim, Y. D.; Arefe, G.; Huang, P. Y.; Lee, C. H.; Chenet, D. A.; Zhang, X.; Wang, L.; Ye, F.; Pizzocchero, F.; Jessen, B. S.; Watanabe, K.; Taniguchi, T.; Muller, D. A.; Low, T.; Kim, P.; Hone, J. Multi-Terminal Transport Measurements of $\mathrm{MoS}_{2}$ Using a van Der Waals Heterostructure Device Platform. Nat. Nanotechnol. 2015, 10, 534-540.

(14) Hishiyama, Y.; Kaburagi, Y. Electrical Resistivity of Highly Crystallized Kish Graphite. Carbon 1992, 30, 483-486.

(15) Moore, A. W. Highly Oriented Pyrolytic Graphite. Chem. Phys. Carbon 1973, 11, 69-187.
(16) Spain, I. L.; Ubbelohde, A. R.; Young, D. A. Electronic Properties of Well Oriented Graphite. Philos. Trans. R. Soc., A 1967, 262, 345-386.

(17) Murakami, M.; Nishiki, N.; Nakamura, K.; Ehara, J.; Okada, H.; Kouzaki, T.; Watanabe, K.; Hoshi, T.; Yoshimura, S. High-Quality and Highly Oriented Graphite Block from Polycondensation Polymer Films. Carbon 1992, 30, 255-262.

(18) Murakami, M.; Yoshimura, S. Highly Conductive Pyropolymer and High-Quality Graphite from Polyoxadiazole. Synth. Met. 1987, 18, 509-514.

(19) Murakami, M.; Watanabe, K.; Yoshimura, S. High quality Pyrographite Films. Appl. Phys. Lett. 1986, 48, 1594-1596.

(20) Kaneka Corp.; http://www.kaneka.co.jp/en/.

(21) Kaburagi, Y.; Hishiyama, Y. Highly Crystallized Graphite Films Prepared by High-Temperature Heat Treatment from Carbonized Aromatic Polyimide Films. Carbon 1995, 33, 773-777.

(22) Murakami, M.; Tatami, A.; Tachibana, M. Fabrication of High Quality and Large Area Graphite Thin Films by Pyrolysis and Graphitization of Polyimides. Carbon 2019, 145, 23-30.

(23) Ohashi, Y.; Koizumi, T.; Yoshikawa, T.; Shiiki, T.; Hironaka, K. Size Effect Inthe Thin Electrical Crystals Resistivity of Very Graphite. Tanso 1997, 180, 235-238.

(24) Venugopal, G.; Kim, S. J. Temperature Dependence of PlanarType Graphite Structures. J. Korean Phys. Soc. 2009, 55, 1102-1105.

(25) Berger, C.; Song, Z.; Li, T.; Li, X.; Ogbazghi, A. Y.; Feng, R.; Dai, Z.; Marchenkov, A. N.; Conrad, E. H.; First, P. N.; de Heer, W. A. Ultrathin Epitaxial Graphite: 2D Electron Gas Properties and a Route toward Graphene-Based Nanoelectronics. J. Phys. Chem. B 2004, 108, 19912-19916.

(26) Tsang, D. Z.; Dresselhaus, M. S. The C-Axis Electrical Conductivity of Kish Graphite. Carbon 1976, 14, 43-46.

(27) Primak, W.; Fuchs, L. H. Electrical Conductivities of Natural Graphite Crystals. Phys. Rev. 1954, 95, 22-30.

(28) Valdes, L. Resistivity Measurements on Germanium for Transistors. Proc. IRE 1954, 42, 420-427.

(29) Muralt, P.; Pohl, D. W. Scanning Tunneling Potentiometry. Appl. Phys. Lett. 1986, 48, 514-516.

(30) Lüpke, F.; Korte, S.; Cherepanov, V.; Voigtländer, B. Scanning Tunneling Potentiometry Implemented into a Multi-Tip Setup by Software. Rev. Sci. Instrum. 2015, 86, 123701.

(31) van der Pauw, L. J. A Method of Measuring Specific Resistivity and Hall Eeffect of Discs of Arbitrary Shape. Philips Res. Rep. 1958, 13, 1-9.

(32) Yoshida, A.; Hishiyama, Y. Electron Channeling Effect on Highly Oriented Graphites-Size Evaluation and Oriented Mapping of Crystals. J. Mater. Res. 1992, 7, 1400-1405.

(33) Kaburagi, Y.; Yoshida, A.; Hishiyama, Y. Microtexture of Highly Crystallized Graphite as Studied by Galvanomagnetic Properties and Electron Channeling Contrast Effect. J. Mater. Res. 1996, 11, 769778.

(34) Zhang, Y.; Small, J. P.; Pontius, W. V.; Kim, P. Fabrication and Electric-Field-Dependent Transport Measurements of Mesoscopic Graphite Devices. Appl. Phys. Lett. 2005, 86, 073104.

(35) Miccoli, I.; Edler, F.; Pfnür, H.; Tegenkamp, C. The 100th Anniversary of the Four-Point Probe Technique: The Role of Probe Geometries in Isotropic and Anisotropic Systems. J. Phys.: Condens. Matter 2015, 27, 223201.

(36) Wasscher, J. D. Note on Four-Point Resistivity Measurements on Anisotropic Conductors. Philips Res. Rep. 1946, 157, 301-306.

(37) van der Pauw, L. J. Determination of Resistivity Tensor and Hall Tensor of Anisotropic Conductors. Philips Res. Rep. 1961, 16, 187-195.

(38) Shiraki, I.; Tanabe, F.; Hobara, R.; Nagao, T.; Hasegawa, S. Independently Driven Four-Tip Probes for Conductivity Measurements in Ultrahigh Vacuum. Surf. Sci. 2001, 493, 633-643.

(39) Albers, J.; Berkowitz, H. L. An Alternative Approach to the Calculation of Four-Probe Resistances on Nonuniform Structures. J. Electrochem. Soc. 1985, 132, 2453-2456. 
(40) Simonis, P.; Goffaux, C.; Thiry, P.; Biro, L.; Lambin, P.; Meunier, V. STM Study of a Grain Boundary in Graphite. Surf. Sci. 2002, 511, 319-322.

(41) Ma, R.; Huan, Q.; Wu, L.; Yan, J. J.; Guo, W.; Zhang, Y.; Wang, S.; Bao, L.; Liu, Y.; Du, S.; Pantelides, S. T.; Gao, H. Direct FourProbe Measurement of Grain-Boundary Resistivity and Mobility in Millimeter-Sized Graphene. Nano Lett. 2017, 17, 5291-5296.

(42) Cummings, A. W.; Duong, D. L.; Nguyen, V. L.; Van Tuan, D.; Kotakoski, J.; Barrios Vargas, J. E.; Lee, Y. H.; Roche, S. Charge Transport in Polycrystalline Graphene: Challenges and Opportunities. Adv. Mater. 2014, 26, 5079-5094.

(43) Yu, Q.; Jauregui, L. A.; Wu, W.; Colby, R.; Tian, J.; Su, Z.; Cao, H.; Liu, Z.; Pandey, D.; Wei, D.; Chung, T. F.; Peng, P.; Guisinger, N. P.; Stach, E. A.; Bao, J.; Pei, S. S.; Chen, Y. P. Control and Characterization of Individual Grains and Grain Boundaries in Graphene Grown by Chemical Vapour Deposition. Nat. Mater. 2011, 10, 443-449.

(44) Tsen, A. W.; Brown, L.; Levendorf, M. P.; Ghahari, F.; Huang, P. Y.; Havener, R. W.; Ruiz-Vargas, C. S.; Muller, D. A.; Kim, P.; Park, J. Tailoring Electrical Transport Across Grain Boundaries in Polycrystalline Graphene. Science 2012, 336, 1143-1146.

(45) Mogi, H.; Wang, Z. H.; Kikuchi, R.; Yoon, C. H.; Yoshida, S.; Takeuchi, O.; Shigekawa, H. Externally Triggerable Optical PumpProbe Scanning Tunneling Microscopy. Appl. Phys. Express 2019, 12, 025005.

(46) Mogi, H.; Wang, Z. H.; Bamba, T.; Takaguchi, Y.; Endo, T.; Yoshida, S.; Taninaka, A.; Oigawa, H.; Miyata, Y.; Takeuchi, O.; Shigekawa, H. Development of Laser-Combined Scanning Multiprobe Spectroscopy and Application to Analysis of $\mathrm{WSe}_{2} / \mathrm{MoSe}_{2}$ in-Plane Heterostructure. Appl. Phys. Express 2019, 12, 045002.

(47) Soule, D. E. Magnetic Field Dependence of the Hall Effect and Magnetoresistance in Graphite Single Crystals. Phys. Rev. 1958, 112, 698-707. 